\title{
Finding and optimising the key factors for the multiple-response manufacturing process
}

\author{
Jeh-Nan Pan ${ }^{\mathrm{a}}$, Jianbiao Pan ${ }^{\mathrm{b}}$ and Chun-Yi Lee ${ }^{\mathrm{a}}$ \\ ${ }^{a}$ Department of Statistics, National Cheng-Kung University, Tainan, Taiwan 70101, Republic of China; \\ ${ }^{b}$ Department of Industrial and Manufacturing Engineering, California Polytechnic State University, \\ San Luis Obispo, CA 93407, USA
}

\begin{abstract}
With the advent of modern technology, manufacturing processes became so sophisticated that a single quality characteristic cannot reflect the true product quality. Thus, it is essential to perform the key factor analysis for the manufacturing process with multiple-input (factors) and multiple-output (responses). In this paper, an integrated approach of using the desirability function in conjunction with the Mahalanobis-Taguchi-Gram Schmit (MTGS) system is proposed in order to find and optimise the key factors for a multipleresponse manufacturing process. The aim of using the MTGS method is to standardise and orthogonalise the multiple responses so that the Mahalanobis distance for each run can be calculated and the multi-normal assumption for the correlated responses can be relaxed. A realistic example of the solder paste stencil printing process is then used to demonstrate the usefulness of our proposed approach in a practical application.
\end{abstract}

Keywords: multiple-response process; solder paste stencil printing process; Mahalonobis distance; desirability function; Taguchi method

\section{Introduction}

With the advent of modern technology, manufacturing processes have become so sophisticated that a single quality characteristic cannot reflect the true product quality. Thus, it is essential to perform the key factor analysis for the manufacturing process with multiple-input (factors) and multiple-output (responses).

A traditional experimental design and analysis method is typically used in finding and optimising the key factors for one response variable. Simultaneous consideration of multiple responses are difficult when (1) these multiple responses are highly correlated, and (2) if one response belongs to 'the bigger the better', while the other belongs to 'the smaller the better' quality characteristic in a multiple-response process. In this paper, we propose a multivariate technique based on the Mahalanobis-Taguchi-Gram Schmit (MTGS) system as well as the desirability function to explore and optimise the key factors for a multiple-response manufacturing process. 


\section{Literature review}

Various multi-response methods have been proposed, for example, the dual response approach proposed by Myers and Carter (1973), the distance function introduced by Khuri and Conlon (1981), the desirability function proposed by Harrington (1965) and modified by Derringer and Suich (1980). Logical solutions to multiple response problems are to optimise overall quality characteristics by compromising between important responses (Myers and Montgomery 1995).

The desirability function is used to reduce the dimension of a multi-response problem into a single-response problem. Derringer and Suich (1980) modified Harrington's (1965) approach and extended to the following three types of quality characteristics:

(1) For the larger-the-better (LTB) type:

$$
d_{i}= \begin{cases}0, & Y_{i} \leq Y_{i^{*}} \\ {\left[\frac{Y_{i}-Y_{i^{*}}}{Y_{i}^{\prime}-Y_{i^{*}}}\right]^{t},} & Y_{i^{*}} \leq Y_{i} \leq Y_{i}^{\prime} \\ 1, & Y_{i} \geq Y_{i}^{\prime}\end{cases}
$$

where $Y_{i^{*}}$ is the minimum acceptable value of $Y_{i}, Y_{i}^{\prime}$ is the highest value of $Y_{i}$.

(2) For the smaller-the-better (STB) type:

$$
d_{i}= \begin{cases}1, & Y_{i} \leq Y_{i}^{\prime \prime} \\ {\left[\frac{Y_{i}^{*}-Y_{i}}{Y_{i}^{*}-Y_{i}^{\prime \prime}}\right]^{r},} & Y_{i}^{\prime \prime} \leq Y_{i} \leq Y_{i}^{*} \\ 0, & Y_{i} \geq Y_{i}^{*}\end{cases}
$$

where $Y_{i}^{\prime \prime}$ is the lowest value of $Y_{i}, Y_{i}^{*}$ is the maximum acceptable value of $Y_{i}$.

(3) For the nominal-the-best (NTB) type:

$$
d_{i}= \begin{cases}{\left[\frac{Y_{i}-Y_{i^{*}}}{c_{i}-Y_{i^{*}}}\right]^{s},} & Y_{i^{*}} \leq Y_{i} \leq c_{i} \\ {\left[\frac{Y_{i}-Y_{i}^{*}}{c_{i}-Y_{i}^{*}}\right]^{t},} & c_{i}<Y_{i} \leq Y_{i}^{*} \\ 0, & Y_{i}>Y_{i}^{*} \text { or } Y_{i^{*}}>Y\end{cases}
$$

where $s$ and $t$ are the exponential parameters that determine the shape of a desirability function, and $c_{i}$ is the most likely acceptable value, $Y_{i^{*}} \leq c_{i} \leq Y_{i}^{*}$, i.e. the target value of the NTB type response. The user has a greater flexibility in the setting up an appropriate desirability function by selecting a suitable $r, s, t$.

After finding the individual desirability function for each response, an overall desirability function can be obtained by

$$
D=\sqrt[k]{d_{1} \times d_{2} \times \cdots \times d_{k}}
$$

where $d_{i}, i=1,2, \ldots, k$ is the individual desirability function for each response. Chiao and Hamada (2001) mentioned that the overall desirability $D=\left(d_{1}\left(Y_{1}\right), d_{2}\left(Y_{2}\right), \ldots, d_{k}\left(Y_{k}\right)\right)^{1 / k}$ 
is difficult to construct if its desirability is complex functions of the $k$ responses, where $\left(Y_{1}, Y_{2}, \ldots, Y_{k}\right)$ are $k$ responses and $\left(d_{1}, d_{2}, \ldots, d_{k}\right)$ are the individual desirability functions. They proposed a quality measure to be the probability that the $k$ responses are simultaneously meeting a specification region for searching the key factors.

Since a traditional multi-dimensional or multivariate system only considers response variables that are mutually independent, it is easy to make mistakes in determining the key factors for the dependent response variables in a multi-dimensional system. Taguchi and Jugulum (2002) proposed a Mahalanobis-Taguchi system (MTS) as shown in Figure 1 to modify the traditional multi-dimensional system.

MTS can be used to detect the abnormality of observations. If the Mahalanobis distance of a data set is too large, then the observation is considered to be abnormal. It should be pointed out that there have been debates on the MTS vs. classical multivariate analysis approaches recently. (For details please refer to Woodall et al. 2003, Jugulum et al. 2003, Abraham and Variyath 2003 and Hawkins 2003).

\section{Research methodology and data analysis procedures}

The data analysis procedures for determining and optimising the key factors of a multiresponse manufacturing process are shown in Figure 2. The determination of key factors for a multi-response manufacturing process is discussed in Section 3.1. The main idea in the data analysis procedures is to use the concept of Mahalonobis distance to convert multi-response variables into a single dimensional performance index in analysis of variance (ANOVA). The optimisation of a multi-response manufacturing process using a desirability function is discussed in Section 3.2.

\subsection{The key factor analysis for a multi-response manufacturing process}

Denote $n$ observations of the $k$ responses by $\boldsymbol{W}_{1}, \boldsymbol{W}_{2}, \ldots, \boldsymbol{W}_{k}$, where $\boldsymbol{W}_{i}=$ $\left(w_{i 1}, w_{i 2}, \ldots, w_{i n}\right)^{\prime}, i=1,2, \ldots, k$. The original data structure for a multi-response process is shown in Table 1.

Step 1: Perform the standardisation.

The original experimental data $\left(w_{i j}\right)$ need to be standardised as below

$$
z_{i j}=\frac{w_{i j}-\bar{w}_{i}}{\sqrt{S_{i}^{2}}}\left\{\begin{array}{l}
i=1,2, \ldots, k \\
j=1,2, \ldots, n
\end{array}\right.
$$

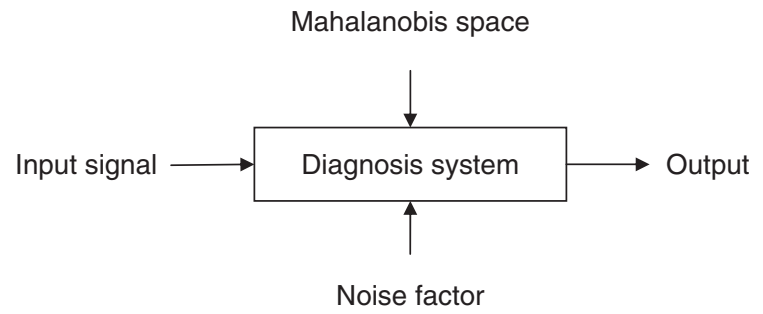

Figure 1. The Mahalanobis-Taguchi multidimensional system. 
Determination of the key factors

(1)

Collect $n$ observations $W_{i}$ for the

$k$-responses, where

$W_{i}=\left(w_{i 1}, w_{i 2}, \ldots w_{i n}\right)^{\prime}, i=1,2, \ldots k$

(2)

(3)

(4)

(5)

Determine the initial key factors for the response $\sqrt{M D_{\mathrm{j}}}$ by effect normal probability and Pareto plots.

(6)

Perform analysis of variance to confirm the significant main and interaction effects.

Perform regression and residual

(7)

regression model.

(8)

Calculate the desirability function for each response under different combinations of key factors

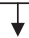

Calculate the overall desirability function under different combinations of key factor levels, i.e. $D=\sqrt[k]{d_{1} \times d_{2} \times \times d_{k}}$.

(10)

Find the maximum overall desirability function that is the optimal combination of key factor levels.

Perform confirmatory tests using the fitted regression model and calculate Mean Absolute Percent Error (MAPE). A MAPE less than $15 \%$ is preferred.

Figure 2. The data analysis flowchart for determining and optimising the key factors of a multi-response process.

Table 1. The original data structure for a multi-response process.

\begin{tabular}{lcccc}
\hline Run $\boldsymbol{j}$ & $\boldsymbol{W}_{1}$ & $\boldsymbol{W}_{2}$ & $\cdots$ & $\boldsymbol{W}_{k}$ \\
\hline 1 & & & & \\
2 & $w_{11}$ & $w_{21}$ & $\ldots$ & $w_{k 1}$ \\
$\vdots$ & $w_{12}$ & $w_{22}$ & $\cdots$ & $w_{k 2}$ \\
$n$ & $\vdots$ & $\vdots$ & $\vdots$ & $\vdots$ \\
\hline
\end{tabular}


where

$$
\bar{w}_{i}=\frac{\sum_{j=1}^{n} w_{i j}}{n} \quad \text { and } \quad S_{i}^{2}=\frac{\sum_{j=1}^{n}\left(w_{i j}-\bar{w}_{i}\right)}{n-1}
$$

Step 2: Perform the orthogonalisation.

According to the orthogonal procedure of Gram-Schmidt process (GSP) described below, the standardised response $Z_{1}, Z_{2}, \ldots, Z_{k}$ can be converted to an orthogonalised response $U_{1}, U_{2}, \ldots, U_{k}$. The orthogonal data for a multi-response process is shown in Table 2.

$$
\begin{aligned}
\boldsymbol{U}_{1}= & \boldsymbol{Z}_{1}=\left(u_{11}, u_{12}, \ldots, u_{1 n}\right)^{\prime} \\
\boldsymbol{U}_{2}= & \boldsymbol{Z}_{2}-c_{2,1} \boldsymbol{U}_{1}=\left(u_{21}, u_{22}, \ldots, u_{2 n}\right)^{\prime} \\
\boldsymbol{U}_{3}= & \boldsymbol{Z}_{3}-c_{3,1} \boldsymbol{U}_{1}-c_{3,2} \boldsymbol{U}_{2}=\left(u_{31}, u_{32}, \ldots, u_{3 n}\right)^{\prime} \\
& \vdots \\
\boldsymbol{U}_{k}= & \boldsymbol{Z}_{k}-c_{k, 1} \boldsymbol{U}_{1}-c_{k, 2} \boldsymbol{U}_{2}-\cdots-c_{k, k-1} \boldsymbol{U}_{k-1}=\left(u_{k 1}, u_{k 2}, \ldots, u_{k n}\right)^{\prime}
\end{aligned}
$$

where $c_{i, j}=\left(\boldsymbol{Z}_{i}^{\prime} \boldsymbol{U}_{j} / \boldsymbol{U}_{j}^{\prime} \boldsymbol{U}_{j}\right)$ are the coefficients of vectors in the Gram-Schmidt process.

Step 3: Compute the Mahalonobis distance.

Let $\boldsymbol{V}_{j}=\left(u_{1 j}, u_{2 j}, \ldots, u_{k j}\right)^{\prime}, j=1,2, \ldots, n$ be the $j$ th run data after standardisation and orthogonalisation, then the Mahalonobis distance for the $j$ th run data can be calculated by

$$
M D_{j}=\mathbf{V}_{j}^{\prime} \mathbf{D}^{-1} \mathbf{V}_{j}
$$

\begin{tabular}{|c|c|c|c|}
\hline Run $\boldsymbol{j}$ Response $\boldsymbol{i}$ & $U_{1}$ & $\boldsymbol{U}_{2}$ & $\ldots$ \\
\hline 1 & $u_{11}$ & $u_{21}$ & $\ldots$ \\
\hline 2 & $u_{12}$ & $u_{22}$ & $\ldots$ \\
\hline$\vdots$ & $\vdots$ & $\vdots$ & $\vdots$ \\
\hline$n$ & $u_{1 n}$ & $u_{2 n}$ & $\ldots$ \\
\hline
\end{tabular}

where $\mathrm{D}$ is a variance-covariance matrix of $\boldsymbol{U}_{1}, \boldsymbol{U}_{2}, \ldots, \boldsymbol{U}_{k}$. Since $\boldsymbol{U}_{1}, \boldsymbol{U}_{2}, \ldots, \boldsymbol{U}_{k}$ are orthogonalised data and they are uncorrelated, $\mathbf{D}$ is denoted by:

$$
\mathbf{D}=\left(\begin{array}{ccc}
\sigma_{U_{1}}^{2} 0 & \cdots & 0 \\
0 & & \vdots \\
\vdots & \ddots & \\
0 & \cdots & \sigma_{U_{k}}^{2}
\end{array}\right)
$$

Table 2. The standardised and orthogonal data for a multi-response process. 
Thus, the Mahalonobis distance for the $j$ th run data in MTGS can be rewritten as:

$$
\begin{aligned}
M D_{j} & =\mathbf{V}_{j}^{\prime} \mathbf{D}^{-1} \mathbf{V}_{j} \\
& =\left(\frac{u_{1 j}^{2}}{\sigma_{U_{1}}^{2}}+\frac{u_{2 j}^{2}}{\sigma_{U_{2}}^{2}}+\cdots+\frac{u_{k j}^{2}}{\sigma_{U_{k}}^{2}}\right), \text { when } \sigma \text { are known. } \\
& =\left(\frac{u_{1 j}^{2}}{S_{U_{1}}^{2}}+\frac{u_{2 j}^{2}}{S_{U_{2}}^{2}}+\cdots+\frac{u_{k j}^{2}}{S_{U_{k}}^{2}}\right), \text { when } \sigma \text { are unknown. }
\end{aligned}
$$

According to Taguchi and Jugulum (2002), one can use $y_{j}=\sqrt{M D_{j}}$ as a single response variable. Following the above three steps the multi-response variables can be reduced into a single dimensional performance index, i.e. Mahalonobis distance.

Step 4: Perform ANOVA and regression analysis.

If one only considers the main and two-factor interaction effects in an experimental design, then the effect model for a multi-factor experiment is

$$
Y_{i j \ldots m l}=\mu+\alpha_{i}+\beta_{j}+\cdots+\tau_{m}+\gamma_{l}+(\alpha \beta)_{i j}+\cdots+(\tau \gamma)_{m l}+\varepsilon_{i j \ldots m l}, \quad\left\{\begin{array}{c}
i=1,2, \ldots, k \\
j=1,2, \ldots, n \\
\vdots \\
m=1,2, \ldots, o \\
l=1,2, \ldots, p
\end{array}\right.
$$

where $\mu$ is the overall mean, $\alpha_{i}$ is the $i$ th treatment effect of the first factor, $\beta_{j}$ is the $j$ th treatment effect of the second factor, $(\alpha \beta)_{i j}$ is the $i$ th and the $j$ th interaction effect of the first and second factors. Similarly, $\tau_{m}$ and $\gamma_{l}$ are the $m$ th and $l$ th treatment effects, $(\tau \gamma)_{m l}$ is the $m$ th and $l$ th interaction effect. The three assumptions for the experimental errors in ANOVA are:

(1) Normality, i.e. the error terms $\varepsilon_{i}$ are normally distributed.

(2) Constant variance, i.e. $\operatorname{Var}\left(\varepsilon_{i}\right)=\sigma^{2}$.

(3) Independence, i.e. the error terms $\varepsilon_{i}$ are mutually independent.

Since we are interested in testing the treatment and interaction effects against zero, the null hypotheses of the treatment and interaction effects can be expressed respectively as:

$$
\left\{\begin{array}{l}
H_{0}: \alpha_{1}=\alpha_{2}=\cdots=\alpha_{k}=0 \\
H_{0}: \beta_{1}=\beta_{2}=\cdots=\beta_{n}=0 \\
\vdots \\
H_{0}: \gamma_{1}=\gamma_{2}=\cdots=\gamma_{p}=0
\end{array}\right.
$$

and

$$
\left\{\begin{array}{ll}
H_{0}:(\alpha \beta)_{i j}=0 & \text { for all } i, j \\
\vdots & \\
H_{0}:(\tau \gamma)_{l m}=0 & \text { for all } l, m
\end{array} .\right.
$$


The main and interaction effects are considered to be significant if the null hypotheses are rejected. Normally, data analysis procedures for finding the key factors include the following five steps:

(1) Determine the initial key factors by using effect normal probability and Pareto plots.

(2) Confirm the key factors using ANOVA, and highlight the significant main and interaction effects.

(3) Construct a fitted regression model.

(4) Perform ANOVA and residual analyses including checking the three assumptions for errors, i.e. normality, independence and constant variance.

(5) Perform confirmatory tests by using the fitted regression model, and calculate mean absolute percent error (MAPE) by

$$
\frac{1}{m} \sum_{i=1}^{m} \frac{\left|y_{i}-\hat{y}_{i}\right|}{y_{i}}
$$

where $y_{i}$ are the actual observations and $\hat{y}_{i}$ are the predicted values. It is assumed that no important factors were omitted from the experiment if the percent contribution due to error is less than 15\% (Ross 1988). Thus, a MAPE less than $15 \%$ is recommended.

\subsection{Searching the optimal combination of key factor levels}

After finding the key factors, the multi-response manufacturing process can be optimised using the desirability functions. Assume that

$$
\boldsymbol{B}_{i j \ldots m l}=\mu+\alpha_{i}+\beta_{j}+\cdots+\tau_{m}+\gamma_{l}+(\alpha \beta)_{i j}+\cdots+(\tau \gamma)_{m l}
$$

where $\boldsymbol{B}_{i j \ldots m l}$ is a function of $\mu, \alpha_{i}, \beta_{j}, \ldots, \tau_{m}, \gamma_{l},(\alpha \beta)_{i j}, \ldots,(\tau \gamma)_{m l}$. The least squares estimators of the parameters in a multi-factor effect model can be found by minimising the sum of squares of the errors from Equation (9), i.e.

$$
\begin{array}{r}
\min _{\mu, \alpha_{i}, \beta_{j}, \ldots, \tau_{m}, \gamma_{l},(\alpha \beta)_{i j}, \ldots,(\tau \gamma)_{m l}} \sum_{i=1}^{k} \sum_{j=1}^{n} \cdots \sum_{l}^{p} \varepsilon_{i j \ldots m l}^{2} \\
=\min _{\mu, \alpha_{i}, \beta_{j}, \ldots, \tau_{m}, \gamma_{l},(\alpha \beta)_{i j}, \ldots,(\tau \gamma)_{m l}}(\boldsymbol{Y}-\boldsymbol{B})^{\prime}(\boldsymbol{Y}-\boldsymbol{B})
\end{array}
$$

The solution to Equation (10) is not unique and depends on the following constraints:

$$
\sum_{i=1}^{k} \alpha_{i}=\sum_{j=1}^{n} \beta_{j}=\cdots=\sum_{l=1}^{p} \gamma_{l}=\sum_{i=1}^{k}(\alpha \beta)_{i j}=\sum_{j=1}^{n}(\alpha \beta)_{i j}=\cdots=\sum_{m=1}^{o}(\tau \gamma)_{m l}=\sum_{l=1}^{p}(\tau \gamma)_{m l}=0
$$

Using the above constraints, the least square estimators

$$
\hat{\mu}, \hat{\alpha}_{i}, \hat{\beta}_{j}, \ldots, \hat{\tau}_{m}, \hat{\gamma}_{l},(\hat{\alpha \beta})_{i j}, \ldots,(\hat{\tau \gamma})_{m l}
$$

to the normal equations can be found. Hence, the predicted values $Y_{i j . . m l}^{\prime \prime}$ is obtained by plugging the above least square estimators into Equation (9). Once the predicted values for different combinations of key factor levels are determined, the individual desirability function proposed by Derringer and Suich (1980) can be 
calculated for each of the $k$ responses. The given engineering specification may be either the larger the better, the smaller the better, or the nominal the best. Finally, the overall desirability function $D=\sqrt[k]{d_{1} \times d_{2} \times \cdots \times d_{k}}$ for $k$ responses is calculated for each different combination of the key factor levels and an optimal combination of the key factor levels can be determined by maximising the overall desirability function.

\section{A comparative analysis using simulation}

The Taguchi method using signal-to-noise ratios $(\mathrm{S} / \mathrm{N})$ is commonly used in finding the key factors for a multi-response process. However, the key factor levels obtained by Taguchi method may not be an optimal one. To compare the two methods, an example of advertising styles (Sharma 1996) is used for illustration. There are three advertising styles in his study, i.e. the humour, sentiment and contrast-oriented. Since the evaluation of an advertising style is also affected by the gender, 12 males and 12 females are randomly selected and divided into three groups. Each group consists of four males and four females. Under the influence of each advertising style, every group member is asked to give two scores on the categories of reliability and degree of information for evaluating the advertising impact. The given scores were ranging from 1 to 10 and the correlation coefficient between the reliability and degree of information for each advertising style were found in a range from 0.6 to 1.0, i.e. the two response values are highly correlated. Performing a multivariate ANOVA (MNOVA), the main effect of advertising style and the interaction effect of advertising style and gender are significant at a $90 \%$ significance level $(\alpha=0.1)$. The observations can be expressed in a matrix form $\mathbf{Y}=\mathbf{X B}+\mathbf{E}$ as shown below, where $\boldsymbol{Y}$ and $\mathbf{E}$ are $24 \times 2$ matrices, $\boldsymbol{X}$ is a $24 \times 12$ matrix, and $\mathbf{B}$ is a $12 \times 2$ coefficient vector of regression.

$$
\left[\begin{array}{ll}
\mathbf{y}_{111} & \mathbf{y}_{211} \\
\mathbf{y}_{112} & \mathbf{y}_{212} \\
\mathbf{y}_{113} & \mathbf{y}_{213} \\
\mathbf{y}_{121} & \mathbf{y}_{221} \\
\mathbf{y}_{122} & \mathbf{y}_{222} \\
\mathbf{y}_{123} & \mathbf{y}_{223}
\end{array}\right]=\left[\begin{array}{llllllllllll}
\mathbf{1} & \mathbf{1} & \mathbf{0} & \mathbf{0} & \mathbf{1} & \mathbf{0} & \mathbf{1} & \mathbf{0} & \mathbf{0} & \mathbf{0} & \mathbf{0} & \mathbf{0} \\
\mathbf{1} & \mathbf{1} & \mathbf{0} & \mathbf{0} & \mathbf{0} & \mathbf{1} & \mathbf{0} & \mathbf{1} & \mathbf{0} & \mathbf{0} & \mathbf{0} & \mathbf{0} \\
\mathbf{1} & \mathbf{0} & \mathbf{1} & \mathbf{0} & \mathbf{1} & \mathbf{0} & \mathbf{0} & \mathbf{0} & \mathbf{1} & \mathbf{0} & \mathbf{0} & \mathbf{0} \\
\mathbf{1} & \mathbf{0} & \mathbf{1} & \mathbf{0} & \mathbf{0} & \mathbf{1} & \mathbf{0} & \mathbf{0} & \mathbf{0} & \mathbf{1} & \mathbf{0} & \mathbf{0} \\
\mathbf{1} & \mathbf{0} & \mathbf{0} & \mathbf{1} & \mathbf{1} & \mathbf{0} & \mathbf{0} & \mathbf{0} & \mathbf{0} & \mathbf{0} & \mathbf{1} & \mathbf{0} \\
\mathbf{1} & \mathbf{0} & \mathbf{0} & \mathbf{1} & \mathbf{0} & \mathbf{1} & \mathbf{0} & \mathbf{0} & \mathbf{0} & \mathbf{0} & \mathbf{0} & \mathbf{1}
\end{array}\right]\left[\begin{array}{cc}
\mu_{1} & \mu_{2} \\
\boldsymbol{\alpha}_{1} & \boldsymbol{\alpha}_{2} \\
\boldsymbol{\beta}_{1} & \boldsymbol{\beta}_{2} \\
\boldsymbol{\alpha} \boldsymbol{\beta}_{1} & \boldsymbol{\alpha} \boldsymbol{\beta}_{2}
\end{array}\right]+\left[\begin{array}{lll}
\boldsymbol{\varepsilon}_{111} & \boldsymbol{\varepsilon}_{211} \\
\boldsymbol{\varepsilon}_{112} & \boldsymbol{\varepsilon}_{212} \\
\boldsymbol{\varepsilon}_{113} & \boldsymbol{\varepsilon}_{213} \\
\boldsymbol{\varepsilon}_{121} & \boldsymbol{\varepsilon}_{221} \\
\boldsymbol{\varepsilon}_{122} & \boldsymbol{\varepsilon}_{222} \\
\boldsymbol{\varepsilon}_{123} & \boldsymbol{\varepsilon}_{223}
\end{array}\right]
$$

where $\boldsymbol{y}_{l i j}=\left(y_{l i j 1}, y_{l i j 2}, y_{l i j 3}, y_{l i j 4}\right)^{\prime}, \quad \boldsymbol{\alpha}_{l}=\left(\alpha_{l 1}, \alpha_{l 2}\right)^{\prime}, \quad \boldsymbol{\beta}_{l}=\left(\beta_{l 1}, \beta_{l 2}, \beta_{l 3}\right)^{\prime}, \quad \boldsymbol{\alpha} \boldsymbol{\beta}_{l}=\left(\alpha \beta_{l 11}, \alpha \beta_{l 12}\right.$, $\left.\alpha \beta_{l 13}, \alpha \beta_{l 21}, \alpha \beta_{l 22}, \alpha \beta_{l 23}\right)^{\prime}, \quad \varepsilon_{l i j}=\left(\varepsilon_{l i j 1}, \varepsilon_{l i j 2}, \varepsilon_{l i j 3}, \varepsilon_{l i j 4}\right)^{\prime}, \quad\left(\varepsilon_{1}, \varepsilon_{2}\right)_{i j k}^{\prime} \sim N_{2}(\mathbf{0}, \mathbf{\Sigma}), \quad l=1,2, \quad i=1,2$, $j=1,2,3, k=1,2,3,4$, and

$$
\sum=\left[\begin{array}{cc}
\sigma_{\mathrm{adv}}^{2} & \operatorname{cov}(\mathrm{adv}, \text { gender }) \\
\operatorname{cov}(\mathrm{adv}, \text { gender }) & \sigma_{\text {gender }}^{2}
\end{array}\right] .
$$

Since $\mathbf{B}$ and $\mathbf{E}$ could be estimated from data, 10,000 times of simulation run were repeated using this model $f(\mathbf{Y} \mid \hat{\mathbf{B}}, \hat{\mathbf{E}})$. We then performed the key factors analysis based on the MTGS and the Taguchi $(\mathrm{S} / \mathrm{N})$ methods. The simulation results are summarised in Table 3. It shows that percent accuracies for advertising style and gender using the 
Table 3. Comparison of the simulation results for MTGS and Taguchi $(\mathrm{S} / \mathrm{N})$ methods.

\begin{tabular}{lrcrrr}
\hline & \multicolumn{2}{c}{ MTGS method } & & \multicolumn{2}{c}{ Taguchi (S/N) method } \\
\cline { 2 - 3 } Factors & Times* & Percent accuracy & & Times* & Percent accuracy \\
\hline Advertising style & 8916 & $89.16 \%$ & & 3080 & $30.80 \%$ \\
Gender & 597 & $94.03 \%$ & & 2119 & $78.81 \%$ \\
Interaction & 9123 & $(100 \%-5.97 \%)$ & & $90 \%-21.19 \%)$ \\
\hline
\end{tabular}

Note: *Number of times a factor has been classified to be significant at a $90 \%$ confidence level. Percent accuracy for the gender $=1-$ false alarm rate since it is not a significant factor by MANOVA.

MTGS method are equal to $89.16 \%$ and $94.03 \%$ respectively, which are more accurate than using the Taguchi method $(30.8 \%$ and $78.81 \%)$. Note that both the MTGS and Taguchi methods can achieve more than $90 \%$ accuracy for the interaction effect. Table 3 shows that the MTGS method is more accurate than the Taguchi method in finding the key factors. Therefore, the MTGS method is suggested for finding the key factors for a multi-response process.

\section{A numerical example of the solder paste stencil printing process}

In this section, we perform the statistical analysis of solder paste stencil printing data (Pan et al. 2004) using an integrated MTGS and desirability function approach.

\subsection{Introduction to the solder paste stencil printing process}

The experiment conducted by Pan et al. (2004) has four quality characteristics, i.e. the solder paste deposited volume, area, height, and transfer ratio. Six input variables/ factors are:

- stencil thickness,

- solder paste type,

- board finish,

- print speed,

- aperture size, and

- aperture shape.

Aperture size and shape are considered to be the blocks. The specifications for the experimental design are listed in Table 4 . There are $2^{4} \times 5 \times 4=320$ levels of combination in this experimental design. The experimental data contained 21,106 observations. For the sake of convenience 'aperture size', 'stencil thickness', 'aperture shape', 'board finish', 'solder paste type', 'print speed', and 'transfer ratio' are abbreviated thus: Pitch, ST, AS, $\mathrm{BF}, \mathrm{SPT}$, PS and TR respectively.

Pan et al. (2004) found the key factors affecting solder deposited volume are aperture size (pitch), stencil thickness (ST), aperture shape (AS), board finish (BF), print speed (PS); Pitch*ST, pitch*BF, ST*AS, ST*SPT, ST*PS, AS*PS, BE*SPT, SPT*PS. 
Table 4. Input variables and their levels for a QFP solder paste stencil printing process.

\begin{tabular}{|c|c|c|c|c|c|c|}
\hline \multirow[t]{2}{*}{ Stencil thickness } & Thickness (mil) & \multicolumn{2}{|c|}{4} & \multicolumn{3}{|c|}{6} \\
\hline & Level & \multicolumn{2}{|c|}{1} & \multicolumn{3}{|c|}{2} \\
\hline \multirow[t]{2}{*}{ Solder paste type } & Type & \multicolumn{2}{|c|}{ Type 3} & \multicolumn{3}{|c|}{ Type 4} \\
\hline & Level & \multicolumn{2}{|c|}{1} & \multicolumn{3}{|c|}{2} \\
\hline \multirow[t]{2}{*}{ Board finish } & Method & \multicolumn{2}{|c|}{ Immersion $\mathrm{Ag}$} & \multicolumn{3}{|c|}{ HASL } \\
\hline & Level & \multicolumn{2}{|c|}{1} & \multirow{2}{*}{\multicolumn{3}{|c|}{$\begin{array}{l}2 \\
8\end{array}$}} \\
\hline \multirow[t]{2}{*}{ Print speed } & Speed (in/sec) & \multirow{2}{*}{\multicolumn{2}{|c|}{$\begin{array}{l}2 \\
1\end{array}$}} & \multirow{2}{*}{\multicolumn{3}{|c|}{$\begin{array}{l}8 \\
2\end{array}$}} \\
\hline & Level & & & & & \\
\hline \multirow[t]{2}{*}{ Aperture size } & Pitch (mil) & 30 & 25 & 20 & 16 & 12 \\
\hline & Level & 1 & 2 & 3 & 4 & 5 \\
\hline \multirow{2}{*}{$\begin{array}{l}\text { Aperture shape } \\
\text { (see Figure } 3 \text { ) }\end{array}$} & Shape & BSQFP & SQFP & QFP1 & & $2 \mathrm{FP} 2$ \\
\hline & Level & 1 & 2 & 3 & & 4 \\
\hline
\end{tabular}

Note: QFP1 represents vertical rectangle and QFP2 represents horizontal rectangle.

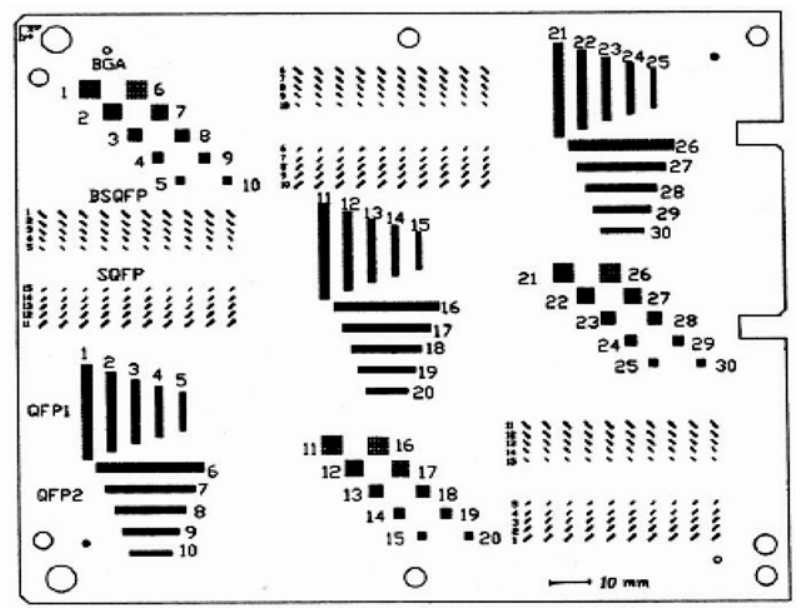

Figure 3. Test pattern.

\subsection{The key factor analysis for the solder paste stencil process}

According to the key factors analysis procedure illustrated in Figure 2, the original multi-response data were first standardised. Then, the GSP was employed to obtain an orthogonal basis for the standardised data, which is used for calculating the Mahalonobis distance of $k$ responses for each run. Finally, $y_{j}=\sqrt{M D_{j}}$ was used as a performance index. It was found that the residuals did not follow a normal distribution. After a Box-Cox transformation with $\lambda=0.15$, the normal assumption is tested to be valid. The ANOVA results for the transformed data are summarised in Table 5. The main effect plot and the two-factor interaction effect plot are shown in Figures 4 and 5, respectively.

Both Figure 4 and Table 5 show that the main factors affecting the QFP manufacturing process are aperture size (Pitch), board finish (BF), solder paste type (SPT). Both Figure 5 
Table 5. ANOVA for QFP data after Box-Cox transformation.

\begin{tabular}{lrrrrrc}
\hline Source & d.f. & Seq. SS & Adj. SS & Adj. MS & F & P \\
\hline Pitch & 4 & 24.03401 & 24.03401 & 6.00850 & 149.53 & 0.000 \\
ST & 1 & 0.06130 & 0.06130 & 0.06130 & 1.53 & 0.218 \\
AS & 3 & 0.17222 & 0.17222 & 0.05741 & 1.43 & 0.235 \\
BF & 1 & 1.11963 & 1.11963 & 1.11963 & 27.86 & 0.000 \\
SPT & 1 & 0.38961 & 0.38961 & 0.38961 & 9.70 & 0.002 \\
PS & 1 & 0.02913 & 0.02913 & 0.02913 & 0.73 & 0.395 \\
Pitch*ST & 4 & 0.44115 & 0.44115 & 0.11029 & 2.74 & 0.029 \\
\hline Pitch*AS & 12 & 0.73673 & 0.73673 & 0.06139 & 1.53 & 0.114 \\
Pitch*BF & 4 & 0.41793 & 0.41793 & 0.10448 & 2.60 & 0.037 \\
Pitch*SPT & 4 & 0.24493 & 0.24493 & 0.06123 & 1.52 & 0.196 \\
Pitch*PS & 4 & 0.47021 & 0.47021 & 0.11755 & 2.93 & 0.022 \\
ST*AS & 3 & 5.78590 & 5.78590 & 1.92863 & 48.00 & 0.000 \\
ST*BF & 1 & 0.85804 & 0.85804 & 0.85804 & 21.35 & 0.000 \\
ST*SPT & 1 & 0.01328 & 0.01328 & 0.01328 & 0.33 & 0.566 \\
ST*PS & 1 & 0.08762 & 0.08762 & 0.08762 & 2.18 & 0.141 \\
AS*BF & 3 & 0.42955 & 0.42955 & 0.14318 & 3.56 & 0.015 \\
\hline AS*SPT & 3 & 0.23467 & 0.23467 & 0.07822 & 1.95 & 0.122 \\
AS*PS & 3 & 0.42388 & 0.42388 & 0.14129 & 3.52 & 0.016 \\
BF*SPT & 1 & 0.41331 & 0.41331 & 0.41331 & 10.29 & 0.002 \\
BF*PS & 1 & 0.11319 & 0.11319 & 0.11319 & 2.82 & 0.094 \\
SPT*PS & 1 & 0.01422 & 0.01422 & 0.01422 & 0.35 & 0.552 \\
Error & 262 & 10.52805 & 10.52805 & 0.04018 & & \\
Total & 319 & 47.01856 & & & & \\
\hline
\end{tabular}

Note: The bold, underlined abbreviation represents significant at $\alpha=0.05$.

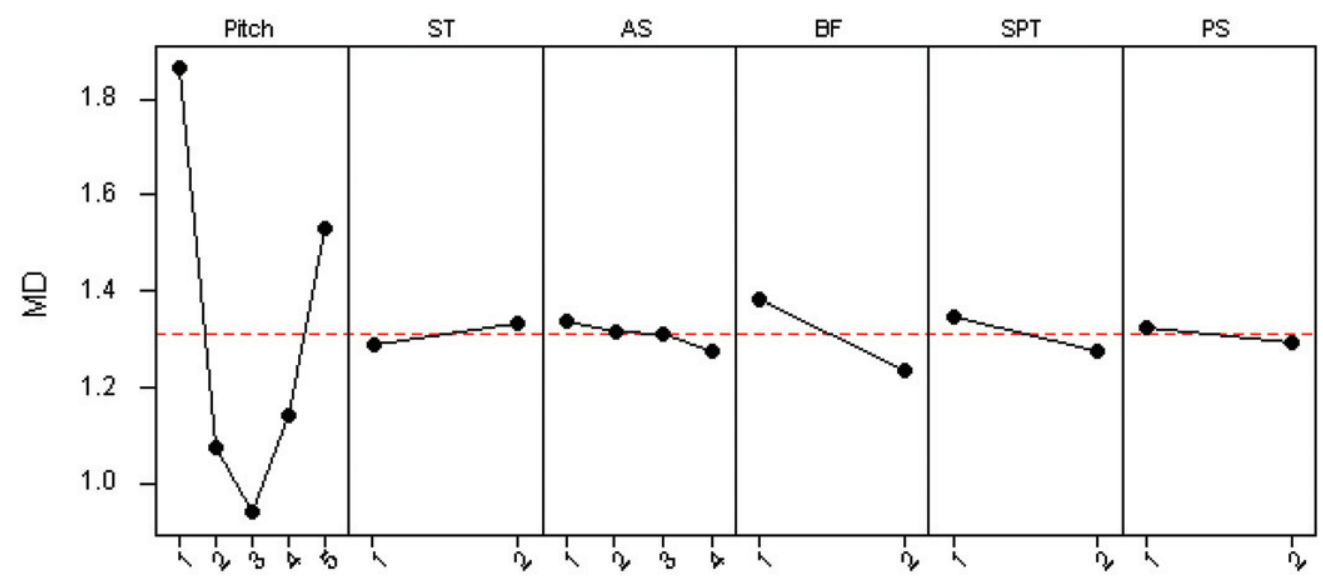

Figure 4. The main effect plots for QFP data after Box-Cox transformation.

and Table 5 show that the significant two-factor interactions are aperture size and stencil thickness (Pitch*ST), aperture size and board finish (Pitch*BF), aperture size and print speed (Pitch*PS), stencil thickness and aperture shape (ST*AS), stencil thickness and board finish $(\mathrm{ST} * \mathrm{BF})$, aperture shape and board finish $(\mathrm{AS} * \mathrm{BF})$, aperture shape and print speed (AS*PS), and board finish and solder paste type (BF*SPT). Adding three 
factors ST, AS, PS, into the above 11 significant factors, a fitted regression equation can be constructed as below:

$$
\begin{aligned}
Y_{t}= & \mu+\sum_{i=1}^{4} \alpha_{i} \operatorname{pitch}_{i}+\beta \times \mathrm{ST}+\sum_{k=1}^{3} \gamma_{k} \mathrm{AS}_{k}+\tau \times \mathrm{BF}+\lambda \times \mathrm{SPT}+\nu \times \mathrm{PS} \\
& +\sum_{i=1}^{4}(\alpha \beta)_{i} \operatorname{pitch}_{i} \times \mathrm{ST}+\sum_{i=1}^{4}(\alpha \tau)_{i} \operatorname{pitch}_{i} \times \mathrm{BF}+\sum_{i=1}^{4}(\alpha \nu)_{i} \operatorname{pitch}_{i} \times \mathrm{PS} \\
& +\sum_{k=1}^{3}(\beta \gamma)_{k} \mathrm{ST} \times \mathrm{AS}_{k}+(\beta \tau) \mathrm{ST} \times \mathrm{BF}+\sum_{k=1}^{3}(\gamma \tau)_{k} \mathrm{AS}_{k} \times \mathrm{BF} \\
& +\sum_{k=1}^{3}(\gamma \nu)_{k} \mathrm{AS}_{k} \times \mathrm{PS}+(\tau \lambda) \mathrm{BF} \times \mathrm{SPT}+\varepsilon_{t}, \\
t= & 1,2, \ldots, 320
\end{aligned}
$$

where $\mu, \alpha_{i}, \beta, \gamma_{k}, \tau, \lambda, \nu,(\alpha \nu)_{i},(\beta \gamma)_{k},(\beta \tau),(\gamma \tau)_{k},(\gamma \nu)_{k}$, and $(\tau \lambda), i=1,2,3,4, k=1,2,3$ are coefficients of the fitted regression model. Table 6 shows that both the main and interaction effects of the regression model are significant. If one wants to estimate the response value for the combination of factors at Pitch $=2, \mathrm{ST}=2, \mathrm{AS}=2, \mathrm{BF}=1$, $\mathrm{SPT}=1, \mathrm{PS}=2$ level, then the predicted value can be obtained from

$$
\hat{Y}=\hat{\mu}+\hat{\alpha}_{1}+\hat{\beta}+\hat{\gamma}_{1}+\hat{v}+(\widehat{\alpha \beta})_{1}+(\widehat{\alpha v})_{1}+(\widehat{\beta \gamma})_{1}+(\widehat{\gamma \nu})_{1}
$$

based on Equation (11).

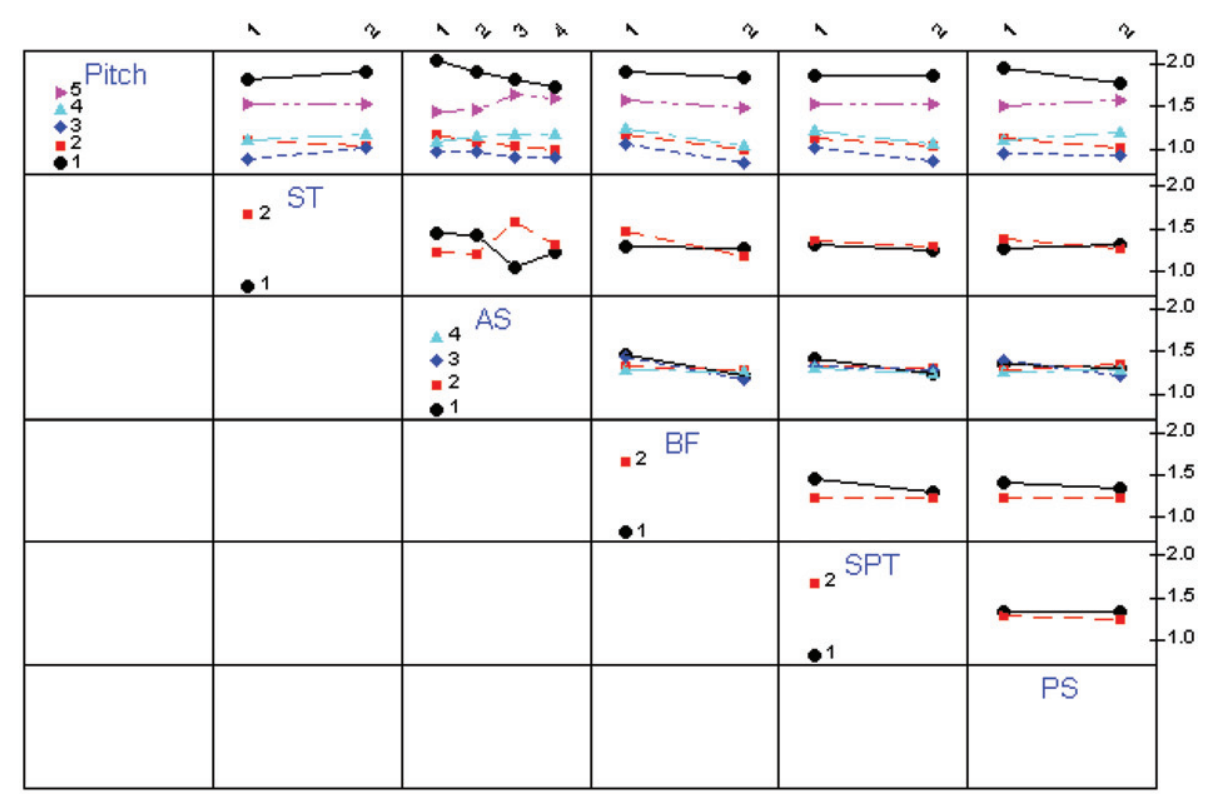

Figure 5. The 2-factor interaction effect plots for QFP data after Box-Cox transformation. 
After checking the validity of the three assumptions of error terms for QFP data after a Box-Cox transformation, the percent contribution for each factor is calculated by:

$$
\rho_{\text {factor }}=\frac{S S_{\text {factor }}^{\prime}}{S S T} \times 100 \%=\frac{S S_{\text {factor }}-d f_{\text {factor }} \times M S E_{\text {pooled }}}{S S T} \times 100 \%
$$

where $S S_{\text {factor }}^{\prime}$ is the pure sum of squares for factors, $S S T$ is the total sum of squares, $S S_{\text {factor }}$ is the sum of squares for factors, $d f_{\text {factor }}$ is a degree of freedom (d.f.) for factor, $M S E_{\text {pooled }}$ is the mean of squares for pooled errors. The significant factors are sorted by the percent contribution and their results are shown in Table 7.

Table 7 indicates that the contributing factors of the solder deposited volume and the transfer ratio for a QFP manufacturing process are aperture size (Pitch) and the interaction of stencil thickness and aperture shape (ST*AS).

\subsection{Using desirability function to find the optimal level of key factors}

To find the optimal combination of key factor levels, we need to specify the quality characteristics target values. In the solder paste stencil printing process, the target values of solder paste deposited volume, area, and height are the volume, area, and height of stencil apertures. The target value of transfer ratio is 1 or $100 \%$. All the four quality

Table 6. The result of ANOVA.

\begin{tabular}{lrrrr}
\hline Source & d.f. & Adj. MS & F & P \\
\hline Main effect & 11 & 2.345991 & 58.387031 & 0.000 \\
Interaction effect & 46 & 0.232274 & 5.780839 & 0.000 \\
Error & 262 & 0.040180 & & \\
Total & 319 & & & \\
\hline
\end{tabular}

Table 7. The percent contribution of the key factors after a Box-Cox transformation.

\begin{tabular}{lrrrr}
\hline Source & d.f. & Seq. SS & Adj. MS & $\begin{array}{c}\text { Percent } \\
\text { contribution (\%) }\end{array}$ \\
\hline Pitch & 4 & 24.0340 & 6.0085 & 50.76 \\
ST*AS & 3 & 5.7859 & 1.9286 & 12.04 \\
BF & 1 & 1.1196 & 1.1196 & 2.29 \\
ST*BF & 1 & 0.8580 & 0.8580 & 1.74 \\
BF*SPT & 1 & 0.4133 & 0.4133 & 0.79 \\
SPT & 1 & 0.3896 & 0.3896 & 0.74 \\
AS*BF & 3 & 0.4295 & 0.1432 & 0.64 \\
Pitch*PS & 4 & 0.4702 & 0.1176 & 0.64 \\
AS*PS & 3 & 0.4239 & 0.1413 & 0.63 \\
Pitch*ST & 4 & 0.4412 & 0.1103 & 0.53 \\
Pitch*BF & 4 & 0.4179 & 0.1045 & \\
Pooled Error & 290 & 12.2353 & 0.0422 & \\
Total & 319 & 47.0186 & & \\
\hline
\end{tabular}


characteristics belong to the nominal-the-best type. The specification limits are required for setting up the desirability function proposed by Derringer and Suich (1980). In this example, we use five-sigma specification limits for the volume, the area, and the height of solder paste deposited and the data are listed in Table 8. The specification limit for the transfer ratio is set to $[0.8,1.2]$ as it is commonly used in the industry. By plugging different key factor levels into the fitted regression Equation (11), one can obtain the predicted values for solder paste deposited volume, area, height, and TR for each combination of key factor levels. Let $t$ and $s$ in Equation (3) equal to 1, the individual desirability functions $\left(d_{1}, d_{2}, d_{3}, d_{4}\right)$ of four responses (i.e. solder paste deposited volume, area, height, and TR) can be calculated for different combinations of key factor levels. The overall desirability function $D=\left(d_{1} \times d_{2} \times d_{3} \times d_{4}\right)^{1 / 4}$ can be achieved by locating the maximum overall desirability function for all the combinations of key factor levels. The optimum combinations of key factor levels for different stencil thicknesses and aperture sizes are shown in Table 9. Note that the levels of each input variables are listed in Table 4. It should be pointed out that the effect of ST and AS cannot be ignored in the optimisation stage although Table 5 shows that ST and AS are not significant main factor. The reason is that their interaction $\mathrm{ST}^{*} \mathrm{AS}$ and $\mathrm{ST} * \mathrm{BF}, \mathrm{AS} * \mathrm{PS}, \mathrm{AS}^{*} \mathrm{BF}$ are significant as shown in Table 5 .

Table 8 . The specification limits of quality characteristics under different combinations of stencil thicknesses and aperture sizes.

\begin{tabular}{|c|c|c|c|c|c|c|c|}
\hline \multirow[b]{2}{*}{ Pitch (mil) } & \multirow[b]{2}{*}{ ST (mil) } & \multicolumn{2}{|c|}{ Volume $\left(\mathrm{mm}^{3}\right)$} & \multicolumn{2}{|c|}{ Area $\left(\mathrm{mm}^{2}\right)$} & \multicolumn{2}{|c|}{ Height (mm) } \\
\hline & & LSL & USL & LSL & USL & LSL & USL \\
\hline \multirow[t]{2}{*}{30} & 4 & 0.039 & 0.119 & 0.476 & 1.104 & 0.060 & 0.140 \\
\hline & 6 & 0.070 & 0.165 & 0.513 & 1.047 & 0.103 & 0.097 \\
\hline \multirow[t]{2}{*}{25} & 4 & 0.011 & 0.099 & 0.252 & 0.847 & 0.048 & 0.152 \\
\hline & 6 & 0.043 & 0.123 & 0.312 & 0.788 & 0.090 & 0.210 \\
\hline \multirow[t]{2}{*}{20} & 4 & 0.009 & 0.069 & 0.112 & 0.668 & 0.052 & 0.148 \\
\hline & 6 & 0.024 & 0.094 & 0.162 & 0.618 & 0.089 & 0.211 \\
\hline \multirow[t]{2}{*}{16} & 4 & 0.004 & 0.048 & 0.057 & 0.463 & 0.049 & 0.151 \\
\hline & 6 & 0.013 & 0.065 & 0.000 & 0.555 & 0.101 & 0.199 \\
\hline \multirow[t]{2}{*}{12} & 4 & 0.000 & 0.038 & 0.000 & 0.339 & 0.000 & 0.210 \\
\hline & 6 & 0.005 & 0.429 & 0.000 & 0.444 & 0.095 & 0.205 \\
\hline
\end{tabular}

Table 9. The optimal combinations of key factor levels under different combinations of stencil thicknesses and aperture sizes.

\begin{tabular}{|c|c|c|c|c|c|c|c|c|}
\hline \multirow[b]{2}{*}{ Pitch } & \multicolumn{4}{|c|}{ ST (4 mil) } & \multicolumn{4}{|c|}{ ST (6 mil) } \\
\hline & AS & $\mathrm{BF}$ & SPT & PS & AS & $\mathrm{BF}$ & SPT & PS \\
\hline 30 & 4 & 1 & 1 & 2 & 3 & 1 & 2 & 2 \\
\hline 25 & 4 & 1 & 1 & 2 & 3 & 1 & 2 & 2 \\
\hline 20 & 4 & 1 & 1 & 2 & 3 & 1 & 2 & 2 \\
\hline 16 & 4 & 2 & 2 & 2 & 3 & 1 & 2 & 1 \\
\hline 12 & 2 & 1 & 1 & 2 & 4 & 2 & 1 & 1 \\
\hline
\end{tabular}


The equation

$$
\frac{1}{m} \sum_{i=1}^{m} \frac{\left|y_{i}-\hat{y}_{i}\right|}{y_{i}}<0.15
$$

is used for calculating the mean absolute percent error (MAPE) after confirmatory tests, where $y_{i}$ are actual observations and $\hat{y}_{i}$ are predicted values. Normally, a MAPE less than $15 \%$ is preferred to ensure that the accuracy of key factors found is greater than $85 \%$. The results in Table 10 show that all the MAPE's of solder paste deposited volume, area, height and transfer ratio (TR) are smaller than 0.15 , which indicate that key factors for the QFP manufacturing process are confirmed.

\section{Discussions}

Most multivariate analysis methods are based on the assumption that multi-responses are mutually independent and follow multi-normal distribution. However, the four responses in our stencil printing process are mutually dependent. To relieve the assumptions of multi-normality and mutual independence among responses, an integrated approach of

Table 10. Summary of the confirmatory tests for the optimal combinations of key factor levels.

Level of the factors

$$
\hat{y}_{i}\left(\frac{\left|y_{i}-\hat{y}_{i}\right|}{y_{i}}\right)
$$

\begin{tabular}{|c|c|c|c|c|c|c|c|c|c|}
\hline Pitch & ST & AS & $\mathrm{BF}$ & SPT & PS & Volume & Area & Height & TR \\
\hline 5 & 1 & 4 & 1 & 1 & 2 & $\begin{array}{c}0.005 \\
(0.084)\end{array}$ & $\begin{array}{c}0.202 \\
(0.126)\end{array}$ & $\begin{array}{c}0.059 \\
(0.011)\end{array}$ & $\begin{array}{c}0.853 \\
(0.106)\end{array}$ \\
\hline 4 & 1 & 4 & 1 & 1 & 2 & $\begin{array}{c}0.019 \\
(0.127)\end{array}$ & $\begin{array}{c}0.325 \\
(0.016)\end{array}$ & $\begin{array}{c}0.076 \\
(0.097)\end{array}$ & $\begin{array}{c}0.997 \\
(0.113)\end{array}$ \\
\hline 3 & 1 & 4 & 1 & 1 & 2 & $\begin{array}{c}0.034 \\
(0.154)\end{array}$ & $\begin{array}{c}0.459 \\
(0.019)\end{array}$ & $\begin{array}{c}0.081 \\
(0.152)\end{array}$ & $\begin{array}{c}1.016 \\
(0.076)\end{array}$ \\
\hline 2 & 1 & 4 & 2 & 2 & 2 & $\begin{array}{c}0.055 \\
(0.092)\end{array}$ & $\begin{array}{c}0.649 \\
(0.029)\end{array}$ & $\begin{array}{c}0.083 \\
(0.049)\end{array}$ & $\begin{array}{c}0.906 \\
(0.003)\end{array}$ \\
\hline 1 & 1 & 2 & 1 & 1 & 2 & $\begin{array}{c}0.083 \\
(0.148)\end{array}$ & $\begin{array}{c}0.893 \\
(0.091)\end{array}$ & $\begin{array}{c}0.086 \\
(0.118)\end{array}$ & $\begin{array}{c}1.051 \\
(0.144)\end{array}$ \\
\hline 5 & 2 & 3 & 1 & 2 & 2 & $\begin{array}{c}0.025 \\
(0.091)\end{array}$ & $\begin{array}{c}0.122 \\
(0.033)\end{array}$ & $\begin{array}{c}0.110 \\
(0.042)\end{array}$ & $\begin{array}{c}0.729 \\
(0.069)\end{array}$ \\
\hline 4 & 2 & 3 & 1 & 2 & 2 & $\begin{array}{c}0.039 \\
(0.002)\end{array}$ & $\begin{array}{c}0.245 \\
(0.023)\end{array}$ & $\begin{array}{c}0.126 \\
(0.004)\end{array}$ & $\begin{array}{c}0.873 \\
(0.043)\end{array}$ \\
\hline 3 & 2 & 3 & 1 & 2 & 2 & $\begin{array}{c}0.054 \\
(0.037)\end{array}$ & $\begin{array}{c}0.379 \\
(0.010)\end{array}$ & $\begin{array}{c}0.131 \\
(0.047)\end{array}$ & $\begin{array}{c}0.893 \\
(0.007)\end{array}$ \\
\hline 2 & 2 & 3 & 1 & 2 & 1 & $\begin{array}{c}0.072 \\
(0.122)\end{array}$ & $\begin{array}{c}0.569 \\
(0.035)\end{array}$ & $\begin{array}{c}0.128 \\
(0.094)\end{array}$ & $\begin{array}{c}0.932 \\
(0.061)\end{array}$ \\
\hline 1 & 2 & 4 & 2 & 1 & 1 & $\begin{array}{c}0.093 \\
(0.120)\end{array}$ & $\begin{array}{c}0.786 \\
(0.043)\end{array}$ & $\begin{array}{c}0.127 \\
(0.047)\end{array}$ & $\begin{array}{c}0.832 \\
(0.120)\end{array}$ \\
\hline MAP & $\sum$ & $\frac{-\hat{y}_{i} \mid}{y_{i}}$ & & & & 0.0977 & 0.0425 & 0.0661 & 0.0742 \\
\hline
\end{tabular}

Note: $y_{i}$ are the actual observations, $\hat{y}_{i}$ are the predicted values, and the values in parentheses represent a MAPE. 
Table 11. ANOVA results using the Taguchi $\mathrm{S} / \mathrm{N}$ method.

\begin{tabular}{lrrrrrr}
\hline Source & d.f. & Seq. SS & Adj. SS & Adj. MS & F & P \\
\hline Pitch & 4 & $292,989.5$ & $292,989.5$ & $73,247.4$ & 164.10 & 0.000 \\
ST & 1 & 36.1 & 36.1 & 36.1 & 0.08 & 0.776 \\
AS & 3 & $19,579.1$ & $19,579.1$ & 6526.4 & 14.62 & 0.000 \\
BF & 1 & 6.0 & 6.0 & 6 & 0.01 & 0.908 \\
SPT & 1 & 4402.8 & 4402.8 & 4402.8 & 9.86 & 0.002 \\
PS & 1 & 90.7 & 90.7 & 90.7 & 0.20 & 0.653 \\
Pitch*ST & 4 & $93,320.0$ & 93320 & 23330 & 52.27 & 0.000 \\
Pitch*AS & 12 & $63,296.1$ & $63,296.1$ & 5274.7 & 11.82 & 0.000 \\
Pitch*BF & 4 & 813.4 & 813.4 & 203.3 & 0.46 & 0.768 \\
Pitch*SPT & 4 & 2443.1 & 2443.1 & 610.8 & 1.37 & 0.245 \\
Pitch*PS & 4 & 767.9 & 767.9 & 192 & 0.43 & 0.787 \\
ST*AS & 3 & 15711 & 15711 & 5237 & 11.73 & 0.000 \\
ST*BF & 1 & 1714.3 & 1714.3 & 1714.3 & 3.84 & 0.051 \\
ST*SPT & 1 & 758.3 & 758.3 & 758.3 & 1.70 & 0.194 \\
ST*PS & 1 & 627.1 & 627.1 & 627.1 & 1.41 & 0.237 \\
AS*BF & 3 & 7041.7 & 7041.7 & 2347.2 & 5.26 & 0.002 \\
AS*SPT & 3 & 8382.5 & 8382.5 & 2794.2 & 6.26 & 0.000 \\
AS*PS & 3 & 5247.4 & 5247.4 & 1749.1 & 3.92 & 0.009 \\
BF*SPT & 1 & 1304.2 & 1304.2 & 1304.2 & 2.92 & 0.089 \\
BF*PS & 1 & 202.9 & 202.9 & 202.9 & 0.45 & 0.501 \\
SPT*PS & 1 & 3446.9 & 3446.9 & 3446.9 & 7.72 & 0.006 \\
Error & 262 & $11,6945.6$ & $116,945.6$ & 446.4 & & \\
Total & 319 & $63,9126.5$ & & & & \\
\hline
\end{tabular}

using the desirability function in conjunction with the MTGS method is proposed to find and optimise the key factors.

We performed another analysis for the same example using the Taguchi $\mathrm{S} / \mathrm{N}$ (signalto-noise) method. Note that the four responses or quality characteristics in our stencil printing process, i.e. the solder paste deposited volume, area, height and transfer ratio belong to 'the nominal-the-best' type. Thus, one can apply the following formula of Taguchi's $\mathrm{S} / \mathrm{N}$ ratio for 'the nominal-the-best' type:

$$
S / N=10^{*} \log _{10}\left(\frac{\bar{Y}^{2}}{S^{2}}\right),
$$

where $\bar{Y}=\sum y_{i} / n, \quad S^{2}=\sum\left(y_{i}-\bar{Y}\right)^{2} /(n-1), \quad y_{i}$ is the quality characteristic. After transforming the four response values into $\mathrm{S} / \mathrm{N}$ ratios under each combination of factor levels, the sum of $\mathrm{S} / \mathrm{N}$ ratios for the four quality characteristics becomes the response value in our ANOVA using the Taguchi method. The ANOVA results using the Taguchi method are shown in Table 11. The ANOVA results from the MTGS method for main factors are compared with the ANOVA results from the Taguchi S/N method and the comparisons are listed in Table 12. It shows that the conclusions drawn from both methods are similar except for AS and BF. Further study is needed to test which method is better in search of the key factors. It should be pointed out that the optimal combinations of key factor levels described in Section 5.3 have not been validated by experiments. 
Table 12. Comparison of the ANOVA results from the MTGS method and the Taguchi method.

\begin{tabular}{lll}
\hline Source & $\begin{array}{c}\text { Using the } \\
\text { MTGS method }\end{array}$ & $\begin{array}{c}\text { Using the Taguchi } \\
\text { S/N method }\end{array}$ \\
\hline Pitch & Significant & Significant \\
ST & Not significant & Not significant \\
AS & Not significant & Significant \\
BF & Significant & Not significant \\
SPT & Significant & Significant \\
PS & Not significant & Not significant \\
\hline
\end{tabular}

Note: Significant at $95 \%$ confidence level.

\section{Summary}

A MTGS method was presented to find and optimise the key factors for a multipleresponse manufacturing process. Detailed procedures of finding and optimising the key factors were described and illustrated with the example of a solder paste stencil printing process. The advantage of this method is that it does not require the assumption of multi-normality and mutual independence among multiple responses.

A comparison of ANOVA results from the MTGS method and the Taguchi $\mathrm{S} / \mathrm{N}$ method was made. The conclusions drawn from both methods are similar though there are some inconsistencies. Further study is needed to explore both the benefits and limitations of the MTGS method.

\section{Acknowledgements}

The authors would like to thank the National Science Council of Taiwan who sponsored this research. Special thanks also go to the editor and two anonymous reviewers for their valuable comments and suggestions.

\section{References}

Abraham, B. and Variyath, A.M., 2003. Discussion. Technometrics, 45 (1), 25-29.

Chiao, C.H. and Hamada, M., 2001. Analysis experiment with correlated multiple responses. Journal of Quality Technology, 33 (4), 451-465.

Derringer, G. and Suich, R., 1980. Simultaneous optimization of several response variables. Journal of Quality Technology, 12 (4), 214-219.

Harrington E.C., Jr, 1965. The desirability function. Industrial Quality Control, 21 (10), 494-498.

Hawkins, D.M., 2003. Discussion. Technometrics, 45 (1), 29-30.

Jugulum, R., Taguchi, G., Taguchi, S. and Wilkins, J.O., 2003. Discussion. Technometrics, 45 (1), $16-21$.

Khuri, A.I. and Conlon, M., 1981. Simultaneous optimization of multiple responses represented by polynomial regression functions. Technometrics, 23, 363-375.

Myers, R.H. and Carter W.H., Jr, 1973. Response surface techniques for dual response systems. Technometrics, 15, 301-317.

Myers, R.H. and Montgomery, D.C., 1995. Response surface methodology: process and product optimization using designed experiments. New York: John Wiley \& Sons, 244-264. 
Pan, J., Tonkay, G.L., Storer, R.H., Sallade, R.M. and Leandri, D.J., 2004. Critical variable of solder paste stencil printing for micro-BGA and fine-pitch QFP. IEEE Transactions on Electronics Packaging Manufacturing, 27 (2), 125-132.

Ross, P.J., 1988. Taguchi techniques for quality engineering. New York: McGraw Hill, 63-200.

Sharma, S., 1996. Applied multivariate techniques. Canada: John Wiley \& Sons.

Taguchi, G. and Jugulum, R., 2002. The Mahalanobis-Taguchi strategy - a pattern technology system. New York: John Wiley \& Sons.

Woodall, W.H., Koudelik, R., Tsui, K.L., Kim, S.B., Stoumbos, Z.G. and Carvounis, C.P., 2003. A review and analysis of the Mahalanobis-Taguchi system. Technometrics, 45 (1), 1-15. 